\title{
Factores clave en la gestión de personas, benchmarking: “Los mejores lugares para trabajar” y Productos Unión
}

\author{
Key factors in people management, benchmarking: "The best places to work" and \\ Union Products
}

\author{
Luis Herrera* \\ EPG. Ciencias Empresariales, Universidad Peruana Unión
}

\section{INFORMACIÓN DEL ARTÍCULO}

Historia del artículo Recibido: julio de 2019 Aceptado: setiembre de 2019

Palabras clave:

Gestión de benchmarking, de personas; credibilidad.

\section{Resumen}

La investigación compara factores clave de gestión de personas en Productos Unión respecto de los Mejores lugares para trabajar utilizando una muestra de 234 trabajadores obtenida por muestreo no probabilístico y en el cual el instrumento global alcanza un alpha de conbrach de 0.961 , el estudio es descriptivo cuantitativo y transversal hallándose que la confianza de los trabajadores en sus mandos está por debajo de los Mejores en $34.34 \%$, la credibilidad $-32 \%$, el respeto $-36 \%$ y la imparcialidad $34 \%$, la experiencia de compañerismo $-32.25 \%$ y el sentimiento de orgullo en $-25.83 \%$, existen diferencias estadísticamente significativas en los promedios de los factores claves de gestión de personas en Productos Unión respecto de los Mejores lugares para trabajar, la integridad y la falta de competencias de los mandos respecto de los Mejores debilita la confianza de los trabajadores, a esto se suma la falta de apoyo y recursos para las tareas, el interés en el trabajador como persona, el trato justo y la equidad en recompensas pero a pesar de la insatisfacción laboral que generan los mandos y los compañeros de trabajo, casi $60 \%$ de los trabajadores considera a Productos Unión como un gran lugar donde trabajar, la calificación no se sustenta en la aplicación de buenas prácticas en la gestión de personas sino en convicciones religiosas, el Señor es el dueño de la Institución y se llega allí para servirle, por eso la lealtad y el orgullo y la razón por lo que existe la oportunidad de obtener mejores resultados organizacionales, de retener y atraer el talento, administrando los factores claves de gestión de personas, basada en motivadores extrínsecos como sucede en los Mejores lugares para trabajar.

The research compares key factors of people management in Products Union with respect to the Best places to work. It uses a sample of 234 workers obtained by non- probabilistic sampling and where the global instrument reaches a conbrach alpha of 0.961 . The study is descriptive, quantitative and transversal. It found that the trust of workers in their leadership is below the Best at $-34.34 \%$, credibility $-32 \%$, respect $-36 \%$ and impartiality $-34 \%$, the experience of fellowship $-32.25 \%$ and the feeling of pride in $-25.83 \%$. There are statistically significant differences in the average of the key factors of people management in Products Union with respect to the Best places to work. The integrity and lack of competences of the leadership regarding the Best, weakens the trust of the workers. 
Keywords:

Key factors of people management; trust; credibility; respect; impartiality; fellowship; pride; Best places to work.
To this is added the lack of support and resources for the tasks, the interest in the work as a person and the fair treatment and fairness in rewards. Despite the job dissatisfaction generated by the managers and co-workers, almost $60 \%$ of the workers consider Products Unión a great place to work. The qualification is not based on the application of good practices in the management of people, but on religious convictions. The Lord is the owner of the Institution and people come there to serve him. This is the reason for loyalty and pride and why there is an opportunity to obtain better organizational results, retain and attract talent, and manage the key factors of people management, based on extrinsic motivators as in the Best places to work.

\section{Introducción}

Reconociendo la importancia del capital humano en la organización, los adventistas del séptimo día han desarrollado indagaciones sobre el clima laboral con resultados de insatisfacción, pero con compromiso en varias investigaciones (Herrera, 2007; Sauco, 2014; Vergara, 2015). Una encuesta que midió la percepción de los trabajadores de la producción en Productos Unión respecto del clima organizacional en el 2014 reveló importantes índices de insatisfacción (Azo, 2014).

Conforme al conocimiento, el desempeño de los colaboradores y los resultados de la organización están condicionados por cómo los individuos perciben su entorno laboral (Marvel, Rodríguez \& Nuñez, 2011), quienes a su vez actúan impulsados por motivaciones extrínsecas, intrínsecas o trascendentes presentes en cualquier acción, aunque con peso distinto (Suarez, 2003). Sabido esto, en el competitivo mundo empresarial "constituirse en un buen lugar para trabajar ha pasado de ser una situación deseable a una creciente necesidad" (Great Place to Work, 2013).

Great Place to Work (ob. cit), se ha dedicado a investigar a nivel mundial por más de 30 años, qué es lo que hace que las personas conciban una organización como un buen lugar para trabajar, sostiene que los Mejores Lugares para Trabajar son el resultado de la gestión de factores claves como: la confianza y sus atributos credibilidad, respeto e imparcialidad; el orgullo y la camaradería (Barbosa, 2012).

Además, personas externas a la organización que han explorado organizaciones las cuales ocupan un lugar en las listas de Los Mejores para Trabajar, han presentado evidencias que estas "disfrutan no sólo de una fuerza laboral estable y positiva sino también de ventajas en rendimiento" (Smithey, Gerhart \& Scott, 2003; Rodríguez, 2012).

Ahora bien, con el propósito de fortalecer los factores claves en la gestión de personas para desarrollar la competitividad organizacional en Productos Unión, subyace la siguiente pregunta de investigación: ¿qué resultados significativos se obtienen en la identificación de los factores claves en la gestión de personas, mediante el benchmarking o evaluación comparativa entre Los Mejores Lugares para Trabajar y Productos Unión?, y en el esquema de la investigación descriptiva cuantitativa y transversal, se estudia la variable simultáneamente en determinado momento haciendo un corte en el tiempo, partiendo de la hipótesis de que existen diferencias significativas en los factores claves de gestión de personas en Productos 
Unión con respecto al promedio de los Mejores Lugares para trabajar en el Perú, se evalúan los resultados significativos que se obtienen en la identificación de los factores claves en la gestión de personas, mediante el benchmarking realizado entre Los Mejores Lugares para Trabajar y Productos Unión.

\section{Materiales y métodos}

Participantes
La unidad de observación son los colaboradores de Productos Unión en el 2016, 317 a nivel nacional. La muestra de 234 trabajadores de la planta de Productos Unión se obtiene por el método de muestreo de tipo no probabilístico, que corresponde al tipo de muestreo por conveniencia.

En la Tabla 1 se visualiza la cantidad de personas encuestadas según el área de trabajo.

Tabla 1

Cantidad de personal encuestado según áreas de trabajo

\begin{tabular}{lcccc}
\hline \multicolumn{1}{c}{ Área } & Frecuencia & Porcentaje & $\begin{array}{c}\text { Porcentaje } \\
\text { válido }\end{array}$ & $\begin{array}{c}\text { Porcentaje } \\
\text { acumulado }\end{array}$ \\
\hline Distribución & 36 & 15,4 & 15,4 & 15,4 \\
\hline Administración & 23 & 9,8 & 9,8 & 25,2 \\
\hline Logística & 9 & 3,8 & 3,8 & 29,1 \\
\hline Calidad/Servicios & 14 & 6,0 & 6,0 & 35,0 \\
\hline Producción & 75 & 32,1 & 32,1 & 67,1 \\
\hline Ventas & 77 & 32,9 & 32,9 & 100,0 \\
\hline Total & 234 & 100,0 & 100,0 & \\
\hline
\end{tabular}

Fuente: Data de cuestionario

\section{Instrumento}

Se usa como cuestionario el Trust Index elaborado por Great Place to Work empleado y aplicado en 50 países, a más de 6,200 organizaciones a nivel mundial y 10 millones de empleados que permite diagnosticar el clima organizacional y comprender lo que hace de un centro de labor un excelente lugar de trabajo.

El cuestionario se entregó a la coordi- nadora de talento humano de la Industria para aplicarlo en reuniones por áreas programadas a través de las gerencias de línea. Terminado cada cuestionario anónimo, fue devuelto a fin de proteger la pertenencia de la información. Para el análisis de los datos se utiliza el software SPSS, en la Tabla 2 se presentan los datos procesados a propósito de la consistencia interna del cuestionario de percepción. 
Tabla 2

Consistencia interna

\begin{tabular}{lcc} 
Dimensión & $N^{\circ}$ de ítems & Alpha \\
& & 0.946 \\
\hline Confianza & 39 & 0.777 \\
\hline Orgullo & 08 & 0.842 \\
\hline Compañerismo & 11 & \\
\hline Total & 58 & 0.961 \\
\hline
\end{tabular}

Fuente: Data, cuestionario de percepción

Ahora bien, en la Tabla 3 se presentan instrumento de recolección de los datos. las dimensiones, indicadores e ítems del

Tabla 3

Dimensiones, indicadores e ítems del instrumento de recolección de datos

\begin{tabular}{|c|c|c|c|}
\hline & \multirow{3}{*}{ Credibilidad } & Comunicación & 7,8,14,19, \\
\hline \multirow{8}{*}{ Confianza } & & Habilidad gerencial & $20,21,25,26,45,57$ \\
\hline & & Integridad & $32,35,41,49$ \\
\hline & \multirow{3}{*}{ Respeto } & Apoyo profesional & $2,9,10,15$ \\
\hline & & Colaboración & 16,22 \\
\hline & & Interés como persona & $3,27,36,43,47,50,53$ \\
\hline & \multirow{3}{*}{ Imparcialidad } & Equidad en recompensas & $4,11,18,52$ \\
\hline & & Ausencia de favoritismo & $23,29,42$ \\
\hline & & Trato justo & $28,33,37,44,46$ \\
\hline \multirow{3}{*}{ Orgullo } & \multicolumn{2}{|c|}{ Trabajo individual } & 12,54 \\
\hline & \multicolumn{2}{|c|}{ Trabajo en equipo } & 5,17 \\
\hline & \multicolumn{2}{|c|}{ Imagen corporativa } & $24,30,38,51$ \\
\hline \multirow{3}{*}{ Compañerismo } & \multicolumn{2}{|l|}{ Familiaridad } & $31,34,40$ \\
\hline & \multicolumn{2}{|l|}{ Hospitalidad } & $1,13,55,56$ \\
\hline & \multicolumn{2}{|c|}{ Sentido de equipo } & $6,39,48,58$ \\
\hline
\end{tabular}

Fuente: Elaboración propia 
Las respuestas del Trust Index elaborado por Great Place To Work que se presentan en porcentajes con respecto al total de la población, están considerando el siguiente significado (Rodríguez, 2012):

Opción 1 y 2: El colaborador no tiene una percepción positiva del aspecto considerado.

Opción 3: El colaborador percibe inconsistencia en el aspecto considerado o se encuentra indeciso o apático.

Opción 4 y 5: El colaborador tiene una percepción positiva del aspecto considerado.

\section{Análisis de datos}

El cuestionario elaborado por Great Place To Work se entregó a la coordinadora del talento humano de Productos Unión para aplicarlo en reuniones por áreas programadas a través de las gerencias de línea. Terminado cada cuestionario anónimo, fue devuelto para asegurar la protección de la información.

Ahora bien, la investigación es descriptiva cuantitativa y transversal por cuanto se estudia la variable simultáneamente en determinado momento haciendo un corte en el tiempo. El benchmarking se desarrolla desde la perspectiva que tiene el trabajador sobre su organización como un todo y se contrasta con el ranking de los $45 \mathrm{Me}-$ jores Lugares para Trabajar en el Perú del 2013

Para el análisis de los datos se utiliza el software SPSS y se opta por utilizar una prueba no paramétrica para la contratación de la hipótesis (comparación de dos muestras independientes), que en este caso es la U Mann Whitney.

\section{Resultados y Discusión}

\section{Resultados 1}

En el modelo de benchmarking un excelente lugar para trabajar en la perspectiva del empleado es el resultado de la satisfacción laboral por la relación del trabajador con sus mandos basada en la confianza; la relación del empleado con sus pares, colegas y compañeros, donde disfruta de la gente con la que trabaja denominada compañerismo o camaradería; y la relación del empleado en su actividad y la firma en donde labora que genera un sentimiento de orgullo "por la labor propia y por el entorno en el que trabaja, a niveles de departamento u organización". (Hernández \& Victoria, 2009, pp. 141).

Ahora bien, la confianza es fundamental para construir un buen lugar para trabajar y uno de los principios de Deming para la gestión y transformación de la eficacia empresarial (Anderson, et al., 1994). Como actitud individual (Acquarone \& Stang, s/f), la confianza es requisito indispensable para quienes lideran personas con el propósito de alcanzar objetivos y producir cambios (Spaemann, 2005), y una condición de trabajo para la productividad en la teoría Z de William Ouchi (García, Posada \& Hernández, 2014). Sin embargo, si podemos visualizar en la figura 1 , la figura de una gaviota, veremos también que el ala más caída en la Industria respecto a los Mejores (-34.34) es precisamente la confianza con un tercio por debajo del promedio de quienes están calificando como marcas empleadoras (Gestión, 2015), es decir donde el talento quiere estar, organizaciones que hoy no solo buscan atraer capacidades que agreguen valor sino también retenerlo y se hacen atractivas alineando "los objetivos 
organizacionales con los objetivos profesionales, personales y sociales de sus empleados" (Tendencias globales en capital humano, 2014).

Es así como, la confianza es un capital social imprescindible en la mano del líder para la consecución de resultados organizacionales, "un recurso fundamental para crear un clima laboral estable y productivo por la sensación de seguridad que establece (Hernández \&Victoria 2009). Mientras que en los Mejores más de $83 \%$ de los colaboradores manifiesta tener confianza en sus líderes, en la Industria menos de la mitad expresa confianza en quienes los lideran. Hay necesidad de que quienes dirigen en la Industria obtengan mayor confianza de sus colaboradores.

De modo que, sobre la plataforma de nuestro modelo donde la confianza es el resultado de la credibilidad depositada hacia los líderes por los colaboradores, el respeto que ellos mismos se hayan ganado y la imparcialidad demostrada en sus relaciones la figura 1 muestra la importancia de desarrollar prácticas de gestión de personas que contribuyan al fortalecimiento de estos atributos de la confianza.

Sin duda alguna, el ala derecha de la gaviota en la figura 1 también dibuja una importante diferencia respecto de los "Mejores". Al igual que la confianza el resultado del compañerismo es un factor crítico con más de un tercio por debajo del promedio de los Mejores (-32.25). En un contexto donde los colaboradores buscan la felicidad en el trabajo (Del Castillo \& Yamada, 2008), un importante $43 \%$ de personas no declara que la industria sea un lugar de compañerismo, y los estudios nos dicen que "el nivel de producción depende de la integración social, pues las personas no actúan de forma aislada sino como miembros de un grupo, y el trabajo es una actividad grupal, donde el comportamiento está condicionado por normas o estándares sociales" (Ramos, et al., 2007. las cursivas son nuestras). Los teóricos han señalado qué si la organización fomenta la relación de compañerismo y "camaradería, el bienestar de los empleados aumenta" (Barranco, 2016. p.9). McClelland como Maslow se refieren a esta relación (Gerais, 2010) como la necesidad humana de afiliación "el deseo de experimentar relaciones interpersonales cálidas y amigables" (Barranco, 2016. p.9). "Ser parte de una comunidad amiga que se preocupa por uno aumenta el compromiso afectivo con la organización y el disfrute de las tareas. El compromiso afectivo ha demostrado estar fuertemente relacionado con la asistencia y el rendimiento en la organización" (Otter, 2010).

Además, en el modelo Great Place to Work el compañerismo o camaradería es el resultado de la práctica de la fraternidad entendida ésta como la experiencia de ser aceptado como soy y la preocupación existente entre unos y otros; la práctica de la hospitalidad: en el sentido de si el lugar físico muestra que piensan en mí y las personas te hacen sentir como en casa; así como la experiencia de sentirse parte de un equipo, en el que siente que colaboran con uno. Siendo así la lectura de la figura 1 estaría mostrando la necesidad de fortalecer prácticas de fraternidad, hospitalidad y sentido de equipo en la Industria.

Es preciso acotar que, las más exitosas organizaciones actualmente consiguen retención y compromiso del talento por estar "enfocadas en ser una marca de la cual los empleados pueden estar orgullosos" (Tendencias globales en capital humano, 2014), propietarias de un propósito inspirador que se traduzca en la razón de estar allí (Sirota, et al. 2006). Cerca de 1,2 millones de empleados de las 52 principales 
compañías de Fortune 1000 encuestados entre el 2001 hasta 2004, reveló que una de las principales causas por la que la motivación de los colaboradores se hace sostenible en el tiempo es el sentimiento de orgullo (Sirota, et.al, 2006). El sentimiento de orgullo en los colaboradores por los resultados de una marca de la que se saben participes del proyecto los hace más productivos (Chafla, 2014, p.9) y de mayor rendimiento (Páez, s/f, p.14).

Según la figura 1 cerca de 7 de cada 10 de los trabajadores de la Industria manifestaron sentir orgullo de servir allí, sin embargo, en los Mejores el orgullo existe en 9 de cada 10, respecto del promedio el valor se cifra en -25.83, es el factor en negativo menos distante de este grupo de tres. En nuestro modelo de benchmarking el orgullo del colaborador se mide por el sentimiento de satisfacción que tiene respecto de su rol particular en la empresa, el mismo que se desarrolla a partir de perci- bir que sus contribuciones son especiales y únicas para la organización; por el sentimiento del colaborador por ser miembro del equipo, por los logros de este y por el sentimiento de las personas frente al papel de la empresa en la comunidad que proviene de su reputación general o en el sector, de su habilidad para satisfacer las necesidades de sus clientes y de su compromiso con la comunidad en la que está localizada (Mercantil, s/f).

La postura de la gaviota con sus dos alas inclinadas dibujadas en la Figura 1, evidencia mayores debilidades en los factores claves de gestión de personas como ser la confianza que sienten los colaboradores hacia quienes los lideran, siendo este el principal factor crítico, y luego la experiencia de compañerismo dentro de la organización. Productos Unión necesita privilegiar prácticas que fortalezcan estos factores para obtener mejores resultados organizacionales.

Figura 2

Comparación de los factores claves confianza, orgullo y compañerismo en Productos Unión respecto de Los Mejores Lugares para Trabajar

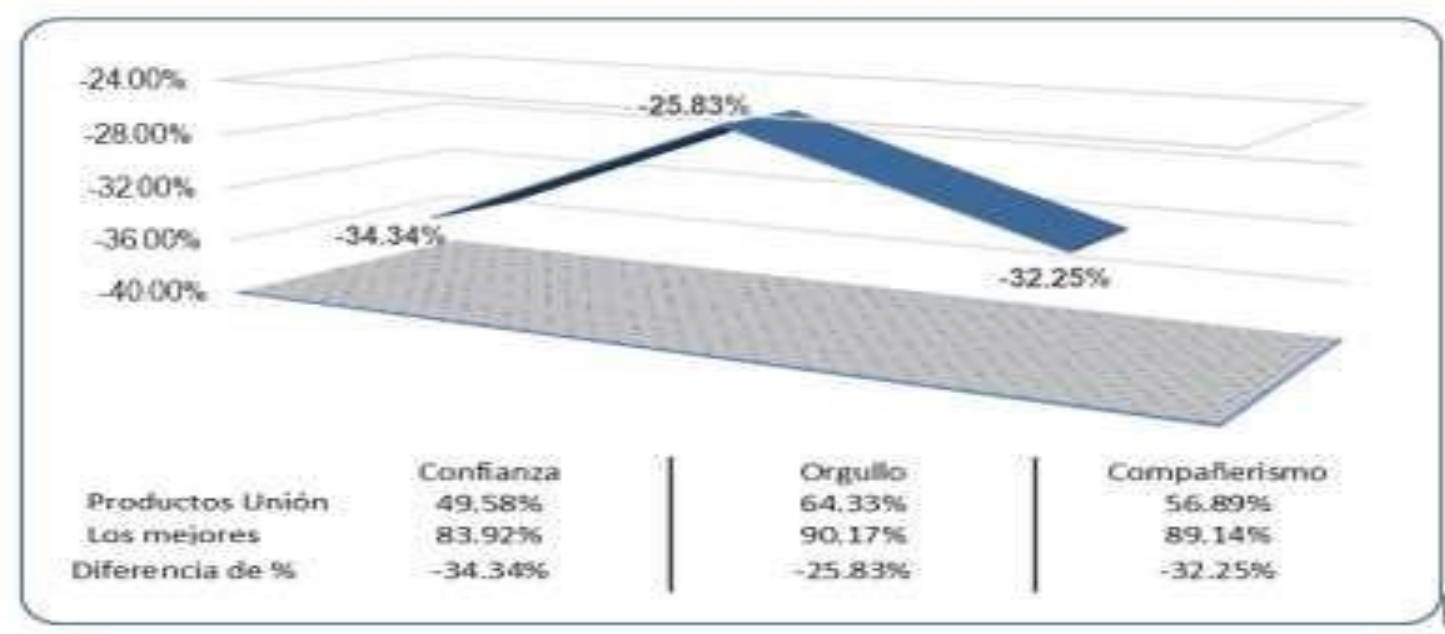

Fuente: Elaboración Propia 


\section{Resultados 2}

En la Figura 2, Figura de la gaviota, se observa el comportamiento de los atributos de la Confianza en Productos Unión respecto de los Mejores lugares para trabajar donde el trato con respeto (observado a través del apoyo profesional, participación o colaboración e interés como persona) es el factor que ocupa la posición más crítica del grupo. A saber, tratar con respeto a los colaboradores es la fuente principal del aumento de la productividad según investigaciones sobre empresas sobresalientes (Gómez, 2015) y la forma como son tratadas las personas aumentan o disminuyen las fortalezas y debilidades de una organización, pueden ser fuente de éxito como de dificultades (Chiavenato, 2009, p.11). Menos de la mitad de los trabajadores de la Industria consideran que existe un trato con respeto de parte de quienes los lideran y respecto del promedio de los Mejores este es el atributo que más debilita la confianza en los líderes.

Otro valor crítico en la Figura 2, es la percepción de imparcialidad en la fábrica (observado en la equidad en recompensas, ausencia de favoritismo y trato justo), más de la mitad de los colaboradores considera que existe un trato parcializado de parte de quienes los lideran. $Y$ un componente básico de la confianza de los empleados en la organización es la imparcialidad porque disminuye la incertidumbre respecto de lo que por actos propios se puede esperar de la organización (Barranco, 2016). Únicamente en condiciones imparciales se obtienen resultados imparciales (Caballero, 2006).

La credibilidad (impulsada por la comunicación, habilidad gerencial y la integridad), es el tercer atributo crítico observable que debilita la confianza en la industria de alimentos, casi la mitad de los colaboradores no le da credibilidad a quienes los dirigen y solamente se es más eficaz persuadiendo cuando se goza de credibilidad (Tormala \& Petty, 2004).

Mientras que en los Mejores arriba del $80 \%$ en promedio de los trabajadores otorga credibilidad a sus líderes, consideran que son tratados con respeto por ellos y perciben que son imparciales, en Productos Unión casi la mitad de ellos no les confiere credibilidad y menos de la mitad considera que son tratados con respeto y que son imparciales.

Para que los líderes puedan obtener la confianza de sus colaboradores es necesario fortalecer prácticas de respeto hacia ellos, de imparcialidad en la organización y desarrollar credibilidad trabajando desde los impulsores de cada uno de estos (figura 2). 
Figura 2

Comparación de los factores claves atributos de la confianza: credibilidad, respeto e imparcialidad, en Productos Unión respecto de los Mejores lugares para trabajar.

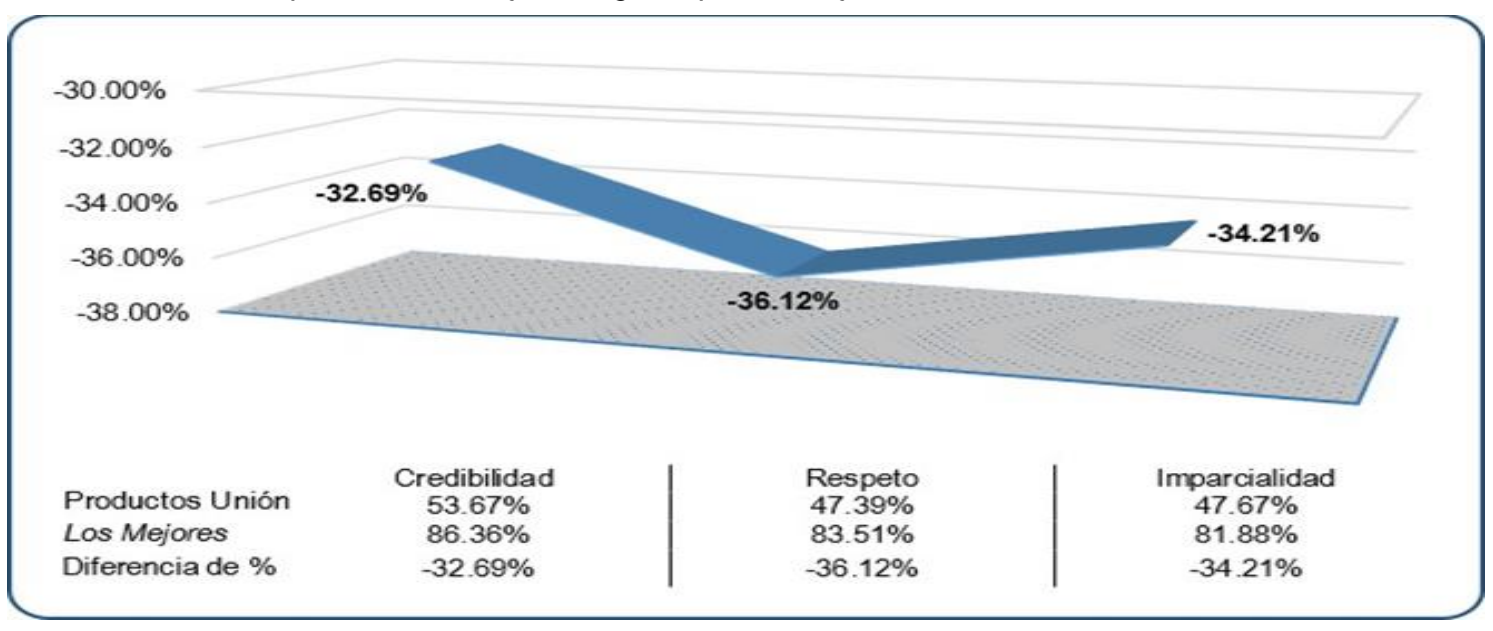

Fuente: Elaboración Propia

Los hallazgos evidencian un problema de fondo como ser la necesidad de mejorar los perfiles de puesto para los mandos y de capacitaciones para las posiciones directivas. Líderes que gocen de la credibilidad de los colaboradores repercutirá positivamente en los resultados organizacionales.

\section{Resultados $3 x$}

Gracias a la aplicación del estadístico no paramétrico U Mann Whitney, se eviden- cia los resultados de los factores confianza, orgullo y compañerismo tienen un coeficiente de 1500.00 ( $p=0.000) ; 0.000$ $(p=0.001)$ y $0.000(p=0.000)$ respectivamente; siendo que el nivel de significancia es menor al 0.05 en los tres factores de comparación, se afirma que existen diferencias significativas en los factores claves confianza, orgullo y compañerismo en Productos Unión respecto de los Mejores lugares para trabajar en el Perú, evidencias a continuación en la Tabla 4.

Tabla 4

Nivel de significancia de los factores claves: confianza, orgullo y compañerismo

\begin{tabular}{lcccc} 
Factores & Productos Unión & Los Mejores & $U$ & Sig. (p-valor) \\
& (rango medio) & (rango medio) & & \\
\hline Confianza & 20.04 & 58.96 & 1500.00 & 0.000 \\
\hline Orgullo & 4.50 & 12.50 & 0.000 & 0.001 \\
\hline Compañerismo & 6.00 & 17.00 & 0.000 & 0.000 \\
\hline
\end{tabular}




\section{Resultados 4}

A razón de visibilizar el Coeficientes de U Mann Whitney de los factores claves de gestión de personas en Productos Unión con respecto a los Mejores Lugares para trabajar en el Perú incluyendo todas sus dimensiones e indicadores se devela lo contentivo como sigue. El resultado obtenido al analizar la hipótesis general, indica que existen diferencias significativas en los factores claves de gestión de personas en Productos Unión con respecto a los Mejores Lugares para trabajar en el Perú. El coeficiente $U$ de Mann Whitney es de 8.000 con un nivel de significancia $p$ de 0.000 , la Tabla 5 afirma la existencia de diferencias entre los factores clave de gestión de personas.

Tabla 5

U de Mann-Whitney $\quad 8,000$

Sig. asintótica (bilateral) $\quad$ 000

a. Variable de agrupación: P. Unión / Los Mejores lugares

\section{Conclusión}

Existen diferencias significativas entre los factores claves para la gestión de personas del Modelo Great Place to Work, la confianza, la credibilidad, el respeto, la imparcialidad, el orgullo y el compañerismo en Productos Unión respecto de los Mejores lugares para trabajar en el Perú $(U$ de Mann Whitney, $p=0.000$ ).

De modo que, la confianza es el factor clave de gestión de personas más crítico en Productos Unión respecto del promedio de los Mejores lugares para trabajar (34.34). Una mitad de las personas en la fábrica no manifiesta confianza en sus mandos y la organización. Al igual que el compañerismo o la camaradería (-32.25), ambos factores tienen resultados de más de un tercio por debajo de los Mejores. Casi la mitad de los trabajadores en la Industria no expresa satisfacción por la relación de compañerismo existente. El sentimiento de orgullo, otro de los factores clave, mantiene igualmente una distancia importante respecto de los Mejores lugares para trabajar más allá de $-25 \%$.

Es así como, la confianza en Productos Unión respecto a los Mejores está debilitada por los factores claves que la alimentan: casi la mitad de los trabajadores en la industria considera que quienes los lideran no son personas de credibilidad (46.33\%), el mayor número de personas sienten que no son tratadas con respeto (52.61\%) y más de la mitad de las personas considera que quienes los dirigen no son imparciales (52.33).

Sin duda alguna, la credibilidad de los mandos (- 32.69) está mermada principalmente por la integridad, menos del $50 \%$ les otorga confiabilidad a sus acciones y palabras. Por sus habilidades gerenciales, más de la mitad observa que en los puestos de trabajo no están las personas adecuadas, cerca del $50 \%$ considera que los líderes carecen de competencias necesarias para la gestión. Y mermada por la necesidad de mejoras en la comunicación, 
casi una mitad de personas percibe que los superiores no están accesibles, y que ellos, los trabajadores no reciben información oportuna incluso sobre cambios que pueden afectar el trabajo.

Ahora bien, el sentimiento en el personal de no ser tratados con respeto (36.12) tiene entre sus principales razones: los beneficios económicos y no económicos que se recibe por su servicio en la organización (interés como persona). El interés de los mandos por su desarrollo profesional (apoyo profesional), el hecho de no tener las facilidades cuando hay necesidad de usar un tiempo libre para atender asuntos de importancia, la inseguridad física de la fábrica para trabajar (interés como persona) y la falta de reconocimiento individual por el buen trabajo y el esfuerzo extra dedicado (apoyo profesional).

Asimismo, la percepción de la imparcialidad de los superiores hacia los trabajadores se traduce, entre otros, principalmente: por no encontrar un trato justo cuando les asiste la razón (trato justo), no recibir una justa recompensa por las ganancias que tiene la organización, no encontrar una justa proporción entre lo que él como trabajador entrega o produce y lo que gana, y observar que los reconocimientos especiales son sólo para unos pocos (equidad en recompensas).

No obstante, el trabajo individual y el trabajo en equipo en Productos Unión son los indicadores que mejor influyen sobre el factor clave orgullo. El $75 \%$ de los trabajadores considera que lo que hacen no es solamente un trabajo, la actividad que desarrollan y a la cual se dedican tiene un significado superior que los llena de orgullo, y que se ve acrecentado por lo que sienten logran además de manera colectiva.
Indudablemente, la relación de camaradería entre pares, colegas y compañeros en la cual el trabajador debe disfrutar de la gente con la que trabaja, muestra importantes necesidades, entre otras, falta de hospitalidad cuando un colaborador llega a un nuevo grupo de trabajo, apoyo de los colegas cuando se les necesita (sentido de equipo), y preocupación por las dificultades personales del colaborador (familiaridad).

Definitivamente, a pesar de la insatisfacción laboral que generan los mandos y las características de relación con los compañeros de trabajo casi $60 \%$ de los trabajadores considera que Productos Unión es un gran lugar donde trabajar. La razón se explica porque detrás de su actividad profesional, productiva, comercial y de negocio, tiene la misión religiosa de compartir su fe y su esperanza (White, 2011e), trabajar aquí es considerado un honor y una responsabilidad de las más sagradas pues es una institución del Señor, él es su dueño, "su influencia y su ejemplo contribuirán mucho para ayudar o para estorbar a la institución en el cumplimiento de su misión" (White, 2011f).

Dicho de otra manera, Productos Unión al ser una dependencia de la Iglesia Adventista del $7 \mathrm{mo}$ día tiene una misión particular que cumplir que la convierte en un gran lugar para trabajar, no es cualquier institución, el dueño es el Señor, se llega allí para servirle. Para la mitad de la población este factor religioso y de misión son suficientes para otorgarle el calificativo de un gran lugar para trabajar al margen de si existen o no buenas prácticas de gestión de personas.

Esta motivación intrínseca que es el motor de los trabajadores no significa que dejen de sentir las carencias existentes que les genera insatisfacción y que ellos 
mismos consideran que deberían corregirse. Las razones para elegir a Productos Unión como un gran lugar para trabajar, no se sustentan en la aplicación de buenas prácticas en la gestión de personas. Por el contrario, los valores porcentuales de las buenas prácticas en los factores claves de gestión de personas se distancian, en el $80 \%$ de los casos, en no menos de un tercio respecto del promedio de los Mejores lugares para trabajar

En suma, los colaboradores de la industria evidencian lealtad y dedicación a la organización a partir de una convicción religiosa, pero con importantes niveles de insatisfacción laboral respecto de los Mejores lugares para trabajar. Los estudios relacionados en otras. Organizaciones adventistas (Herrera, 2007; Sauco, 2014; Vergara, 2015) en los cuales se encontraron resultados parecidos de acuerdo a estos hallazgos obedecerían también a la necesidad de una administración más técnica del capital humano.

Finalmente, un manejo más técnico del capital humano que sume a la ya existente motivación intrínseca las comprobadas ventajas de una buena administración de los factores clave de gestión de personas, basada en motivadores extrínsecos, como sucede en los Mejores lugares para trabajar redundará no sólo en una mayor satisfacción laboral de los colaboradores, sino también en la retención y atracción del talento y por ende en alcanzar mejores resultados organizacionales.

\section{Recomendaciones}

Considerando que la presente investigación es un trabajo de benchmarking, se sugiere trabajar de manera puntual en la influencia de la fe adventista como fuente de motivación intrínseca.

Además, es preciso elaborar, implementar y sostener estrategias de motivación extrínseca, enfocadas en atender los factores claves de gestión de personas de acuerdo al modelo Great Place to Work, priorizando los indicadores críticos como ser el tema: remuneraciones, compensaciones, incentivos, ambiente físico, herramientas o equipos de trabajo, trato, capacitaciones, comunicación y liderazgo.

Finalmente, se exhorta e invita a la Gerencia de Productos Unión a aplicar cuestionarios a fin de realizar benchmarking sobre los factores clave de gestión de personas en otras instituciones patrocinadas por la Iglesia Adventista del Séptimo Día. 


\section{Referencias}

Acquarone, M., \& Stang Cesari, F. (s.f) La confianza como conductor de los capitales empresariales. Buenos Aires, Argentina. Recuperado de: http://www.edutecne.utn.edu.ar/coini_20 13/trabajos/COB18_TC.pdf

Anderson, John C; Rungtusanatham, Manus; Schroeder, Roger G (1994) A theory of quality management underlying the Deming management method. Academy of Management. The Academy of Management Review; Julio; Vol.19, número 3; ProQuest Central. pp. 472509. Universidad Minnesota

Azo, Angi (2014). Informe Clima Organizacional, Productos Unión, área de producción. Recursos Humanos, 11 de junio. Universidad Peruana Unión.

Barbosa, Gomez \& Laura Marcela (2012) Trabajo de investigación sobre los Mejores lugares para trabajar en Colombia. Colegio de Estudios Superiores de Administración. CESA. Trabajo de Grado. Facultad de Administración. Bogotá, DC.

Barranco Fernández, A. (2016). Clima organizacional de un gran lugar para trabajar. ¿Depende del departamento de Recursos Humanos?. ICADE Business School - Universidad Pontificia Comillas ICADE-ICAI

Caballero, J. F. (2006). La teoría de la justicia de John Rawls. Voces y contextos, 2(1), 1-22. Ibero Forum

Chafla Bonilla, V. A. (2014). "Gestión del talento humano y el cumplimiento de la legislación laboral vigente ILA SA". Universidad Técnica de Ambato. Facultad de Contabilidad y Auditoría, Dirección de Posgrado. Ambato, Ecuador
Chiavenato, Idalberto (2009) Comportamiento organizacional. La dinámica del éxito en las organizaciones. Segunda edición. McGraw-Hill/Interamericana Editores, S.A. de C.V. Mexico.

Chiavenato, Idalberto (2009) Gestión del Talento Humano. 3ra ed. Mc Graw Hill. México Del Castillo, Elsa \& Yamada, Gustavo (2008) Responsabilidad social y buen clima laboral: Una fórmula ganadora. Documento de discusión. Marzo, pp. 65-71, Centro de Investigación de la Universidad del Pacífico

García A., I. (2014) Fundamentos del pensamiento administrativo. Escuela de posgrado, Doctorado en Administración. Enero. Universidad Peruana Unión

García G, M., Posada V, N. \& Hernández R, C. (2014) La motivación y los sistemas de recompensas y su impacto en la producción. Contribuciones a la economía, Vol 7

Gerais C., Elías J. (2010) Alineación de los valores del personal de empresas privadas venezolanas de acuerdo con el modelo de gestión basada en valores de Richard Barrett. Universidad Católica Andrés Bello, Facultad de Ciencias económicas y sociales. Noviembre. Caracas, Venezuela. p. 48.

Gestión (2015) Marca Empleadora: ¿Qué gana la empresa y el trabajador con esta estrategia?, 20 de agosto. Empleo y Management. Recuperado de: http://gestion.pe/empleomanagement/marca-empleadora-quegana-empresa-y- trabajador-estaestrategia-2140536

Gomez Galdon, M. V. (2015). Propuestas para una conciliación corresponsable de la vida personal, familiar y laboral. Universitat de València, pp. 1-531 
Great Place To Work (2012) Beneficios de ser un Excelente Lugar para Trabajar. 28 Congreso Internacional de Marketing Financiero. Yacht Club Puerto Madero, Argentina, setiembre. Recuperado:http://es.slideshare.net/am banet/cules-son-los- beneficios-de-serun-excelente-lugar-para-trabajar

Great Place To Work (2013) Recuperado de: http://www.greatplacetowork.com.pe Great Place to Work (2014) Recuperado: http://www.greatplacetowork.com.pe/nu estro-enfoque/icuales-son-los-beneficios

Great Place To Work (2015). Lo que hace un Great Place to Work. Recuperado de http://previous.greatplacetowork.com.pe /gptw/quehacemos.php

Hernández, A. M. \& Victoria, M. E. (2009). La confianza en las organizaciones y la comunicación estratégica: Una experiencia desde la consultoría de Great Place to Work ${ }^{\circledR}$ Institute de México. Comunicación Estratégica: Nuevos horizontes de estudio, 137.

Herrera, R., Luis E. (2007) "Los Mejores Empleadores" y ADRA PERÚ: Benchmarking para la gestión de personal. Universidad Peruana Unión, Escuela de Postgrado. Unidad de Postgrado de Administración

Marvel, M., Rodríguez Monroy, C., \& Núñez Bottini, M. (2011). La productividad desde una perspectiva humana: Dimensiones y factores. Intangible Capital, 7(2), 549-584.

Mercantil, (s.f). Clima Organizacional Mercantil, Conceptos y Resultados. Recuperado:

https://issuu.com/mercantilbanco/docs/cl ima_organizacional_mercantil_concepto s_resultado/9

Otter, Aart den (2010) Authentic Leadership in Great Places to Work. University of Amsterdam. Faculty of Economy and Organizational Studies - Department Business Studies, Master Business
Studies-ip. Holanda

Páez, J. E. G (s/f) La motivación del personal operativo de las plantas industriales y su influencia en la productividad.

Ramos, L. Gloria Enith \& Triana, G. Martha Lucía (2007) Escuela de relaciones humanas y su aplicación en una empresa de telecomunicaciones. Scientia et Technica año XIII, No 34, Mayo. Universidad Tecnológica de Pereira. ISSN 0122-1701, pp 309-314

Rodríguez Lozano, G. (2012). La incidencia del ambiente laboral de las organizaciones sobre el indicador Ebitda: estudio basado en el ranking de las mejores empresas GPTW (20042010) en Colombia. Universidad EAN, Administración de Empresas, octubre, Bogotá DC.

Sauco, H.; Flor de María (2014) "Satisfacción laboral y compromiso institucional en los docentes de las instituciones educativas adventistas de la Misión Ecuatoriana del Sur, 2014". Universidad Peruana Unión, Escuela de posgrado. Unidad de posgrado de Administración

Sirota, David; Mischkind, Louis. \& Meltzer, Michael (2006) Why Your Employees Are Losing Motivation. Working Knowledge, The thinking that leads. Harvard Business School. Recuperado de: http://hbswk.hbs.edu/archive/5289.html

Smithey F. Ingrid; Gerhart Barry \& Scott Kimberly (2003) Are the 100 Best Better? An emperical investigation of the relationship between being a "Great Place to Work" and firm performance. Personnel Psychology. ABI/INFORM Global, pp 965-993

Spaemann, R. (2005). Confianza. Revista empresa y humanismo, 9(2), 131-148. Suarez, L. (2003) Administración de Recursos Humanos. Programa de 
Maestría en Administración de Negocios. Universidad Peruana Unión. Material de clase.

Tendencias Globales en Capital Humano (2014) Deloitte University Press, Deloitte Development LLC. Reino Unido.

Tendencias Globales en Capital Humano (2015) Deloitte University Press, Deloitte Development LLC. Reino Unido.

Tendencias Globales en Capital Humano (2016) Deloitte niversity Press, Deloitte Development LLC. Reino Unido.
Tormala, Z.L. \& Petty, R.E. (2004) Source Credibility an Attitude Certainty: A MetacognitiveAnalysis of Resistance to Persuasion. Journal of Consumer Psychology, 14(4), 427-442

Vergara, C. F. M (2015). Satisfacción laboral y compromiso organizacional en empleados de una universidad privada de Lima. Revista Científica de Ciencias de la Salud, 6(1). 